\title{
Research of Image Detection Method by Computer Vision for Photon Devices Circuit Measurement
}

\author{
Yaqi $\mathrm{Fu}^{1}$,Jiandong $\mathrm{Shi}^{1,2}$,Yaohua Deng ${ }^{1}$, Liming $\mathrm{Wu}^{1}$ \\ (1.Guangdong University of Technology; Guangdong Guangzhou 510006; \\ 2.Sunblence Technologies Co., Ltd;Guangdong Foshan 528000) \\ ( ${ }^{\star}$ Correspond author,Email: 15527297760@163.COM)
}

Key words: Photon device line; Image Detection; Line-width; Radius of curvature

\begin{abstract}
Considering the low efficiency of manual detection method in photon devices fault checking, a new detecting method by image processing for photon device is proposed in this paper, through the new method, photon device images are captured by camera which is installed on microscope, filtering, mosaic, segmentation of images and curvature radius measurement are done by computer, finally, complete circuit diagram of photon devices and the necessary parameter values such as radius of curvature are got. Experiments show that the test results error is $\pm 0.03 \mathrm{um}$, the method can improve production efficiency, shorten the production period and reduce production cost.
\end{abstract}

\section{Introduction}

In recent years, in the field of optical sensing and optical communication, the great potential of photonic integration has received widespread consensus. People in the photonic integration efforts, will make photonic devices or systems in the compactness, cost, performance, greatly improved production $^{[1]}$. Although the device has been detected photons certain research and development, however, mostly manual work, faced with inefficiency, long working hours, and other issues, this paper these problems image detection photonic devices line research methods.

\section{Principle frame of image detection for photon device line}

Photonic devices line image detection method based on a series of image processing technology to realize the automatic detection device line width, and the radius of curvature of intelligent computing device. The method mainly includes the following process, as shown in Fig. 1 .

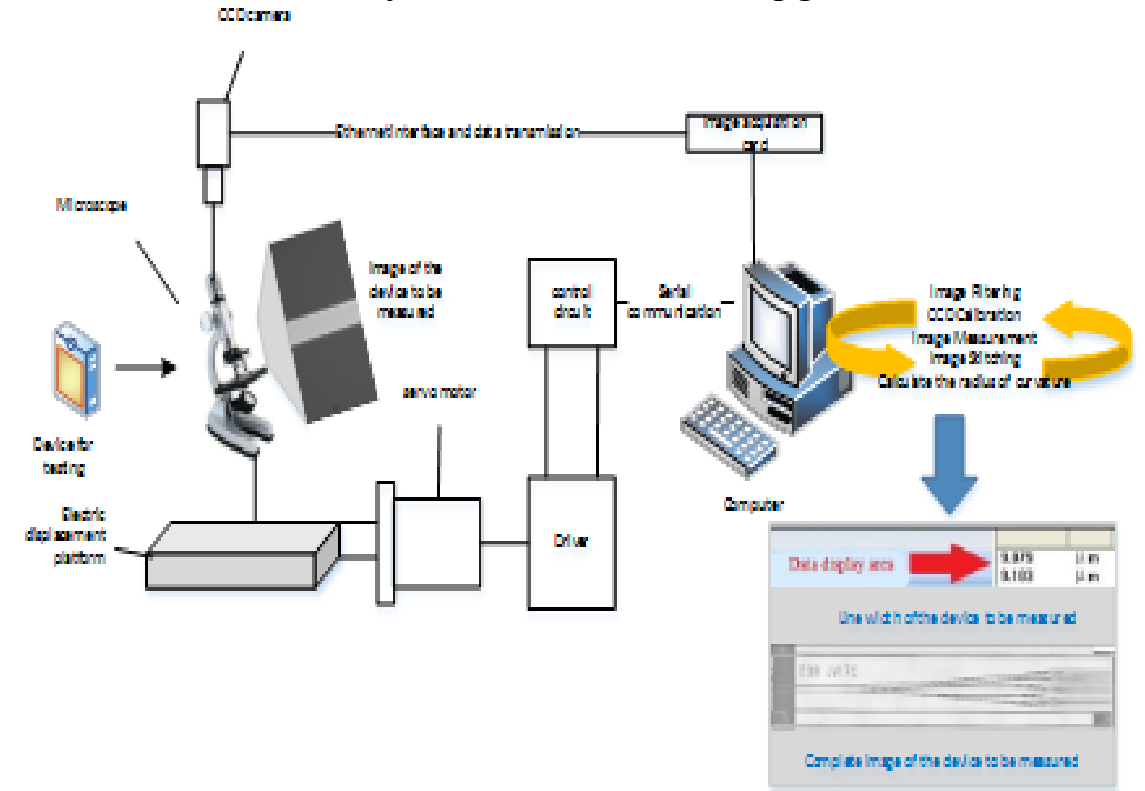

Fig.1 Photonic devices line image detection framework 


\section{Principle description of detection method}

Due to a bad environment with mechanical vibration and other factors, CCD image signal forming, transmission and quantization process will produce a variety of noise. Median filtering is a typical nonlinear filtering method, which depends on the template to achieve. Median filter has good edge features, it is necessary to precise edge localization. Therefore, in this paper, the median filtering method is adopted to filter the image.

CCD Calibration is to determine the relationship between the internal and external parameters of the camera, that is to determine the geometrical and optical characteristics of the camera (internal parameters) and the coordinates of the camera in the three-dimensional world (external parameters) ${ }^{[2]}$ to achieve the conversion between the pixel distance and the actual distance. In this paper, we use the open computer vision function library (OpenCV) to realize the camera calibration.

That is to measure the width of the wave-guide in the photonic device. The region of interest on the first do after the Canny edge detection and Hough linear transformation and displaying the detected straight last two lines of the pixel distance calculation, and the calibration of pixels and the actual distance transformation and actual width is obtained.

\section{Line radius of Curvature of Photonic Devices Derivation}

This article based on SURF algorithm to realize image stitching. Process is as follows:(1)SURF approximate Hessian matrix used to detect the point of interest, and using the integral image ${ }^{[3]}$ significantly reduced the amount of computation. For the image $I$, the Hessian matrix of the $X=(x, y)$ in the $\sigma$ of the point $X$ is defined as: $H(X, \sigma)=\left[\begin{array}{cc}L_{x x}(X, \sigma) & L_{x y}(X, \sigma) \\ L_{x y}(X, \sigma) & L_{y y}(X, \sigma)\end{array}\right]$ (7) where, $L_{x x}(X, \sigma)$ representation of the two order partial derivative of the Gauss in the $X$ and the image $I . L_{x y}(X, \sigma), L_{y y}(X, \sigma)$ has a similar meaning. The approximate template and the results of image convolution with $D_{x x} 、 D_{y y} 、 D_{x y}$ said, using them instead of $L_{x x}, L_{y y} 、 L_{x y}$ to get the approximate Hessian matrix $H_{\text {approx }}$, the determinant is: $\operatorname{det}\left(H_{\text {approx }}\right)=D_{x x} D_{y y}-\left(w D_{x y}\right)^{2}(8)$, where, $w$ is a weight factor, the actual application of the 0.9 can be.(2)Literature [4] points out, SURF algorithm has an important by-product in the process of detection points of interest - brightness contrast identifier. In order to detect a point of interest, through the approximation template convolution integral image, constructed out approximate Hessian matrix and then calculate the determinant of the Hessian matrix constructed on the response graph scale $\sigma$,so that by looking inside the local maximum scale space to locate the point of interest.

\section{Segmentation and Radius of Curvature of the Image Derived Formula}

After completing the image mosaic image of the entire device was, but only line image is needed, so use an image segmentation technique to separate wiring diagram. Since the gray-scale image as a single image, so this take maximum entropy segmentation method ${ }^{[5]}$, as follows: set with $J$ gray-scale image, gray-scale values range for $[0, J-1], p_{j}$ to gray-scale $J$ appears the probability, the information entropy is defined as:

$H=-\sum_{j=0}^{J-1} p_{j} \ln p_{j}(9) t$ will set the threshold for the target image is divided (A) and background (B) two categories, so that the probability distribution of two types $P_{t}=\sum_{j=0}^{t} p_{j}, H_{t}=-\sum_{j=0}^{t} p_{j} \ln p_{j}$ and $\frac{p_{0}}{p_{t}}, \frac{p_{1}}{p_{t}}, \ldots \frac{p_{t}}{p_{t}}, \frac{p_{t+1}}{1-p_{t}}, \frac{p_{t+2}}{1-p_{t}}, \ldots \frac{p_{J-1}}{1-p_{t}}$ respectively, related to the distribution of entropy $H_{A}(t)$ and $H_{B}(t)$.The total entropy of the image is $H_{A}(t)$ and $H_{B}(t)$ is 
$H(t)=\ln p_{t}\left(1-p_{t}\right)+\frac{H_{t}}{p_{t}}+\frac{H-H_{t}}{1-p_{t}}(10)$, and the optimal threshold $t^{*}$ is the segmentation threshold $t^{*}=\operatorname{Arg} \max H(t)(11)$, which makes the maximum value of the total entropy value.

\section{Calculate the radius of curvature}

Curvature radius of curvature is the inverse of, mainly used to describe the degree of a point on the curve of bending. First corner detection was carried out on the segmentation of the image, get straight and curved wave-guide cut-off (arc), determine the scope of the curved wave-guide (arc), then in the bend wave-guide (arc) within the scope of the coordinates of three points. Because the curved wave-guide corresponds to an arc, and each point on the circle is the same degree bend, so the radius of curvature of the circle is the radius of the circle. According to this principle combined with the geometric coordinates of three points (arcs) obtained in said step of bending the wave-guide, can be substituted into circular equation: $(x-a)^{2}+(y-b)^{2}=r^{2}$, where, $x, y$ is the above-mentioned three the coordinates of the point, $a, b$ for the center coordinates, $r$ is the radius.

\section{Testing Laboratory}

Take the optical wave-guide chip as an example, the above method and the analysis of test data measured.

Wave-guide bend into the straight wave-guide and wave-guide boundary straight wave-guide for two parallel lines, the line width is the width measurement measuring optical wave-guide chip straight wave-guide. Fig. 2 shows the measurement area, and Fig. 3 shows the measured results.

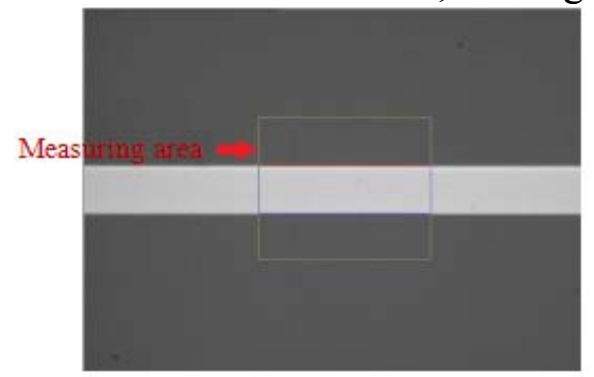

Fig.2 select the measurement area

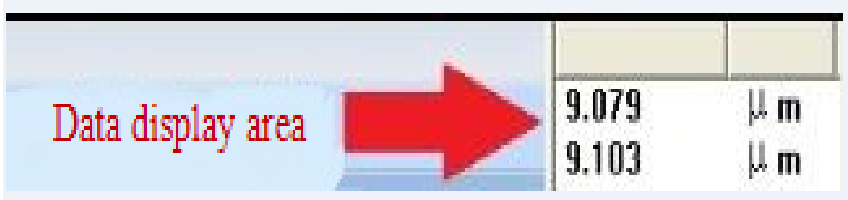

Fig.3 shows the measurement results

As shown in Fig. 2 and Fig.3, when using the mouse to select the measurement area, two parallel lines (red line, blue line) are detected in the measurement area, and the actual line width distance can be seen in the display area after selecting the measurement line width. After interest point detection, interest point description, interest point matching, RANSAC algorithm to eliminate the error matching points can be obtained after the complete device circuit diagram, as shown in Fig.4.

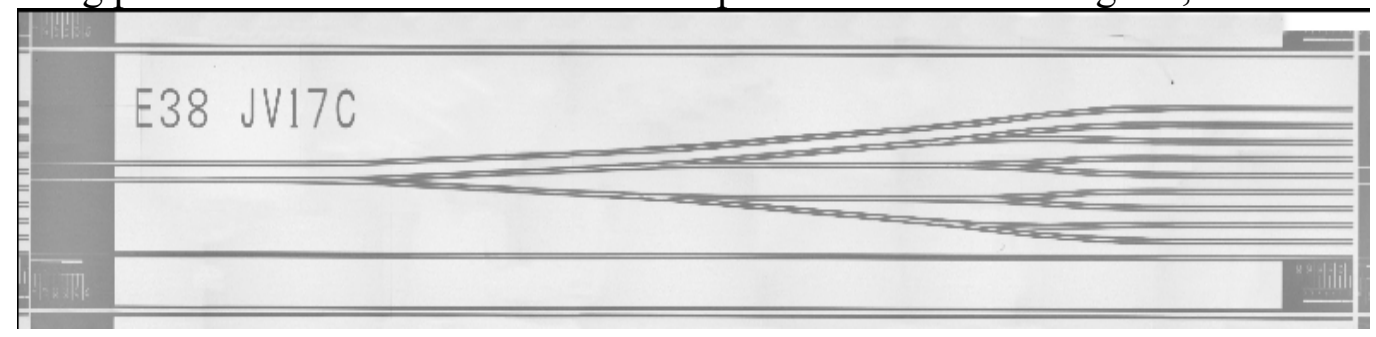

Fig.4 chip complete line distribution 
The experimental results obtained from the test, the method can reach the optical parameters of the effect detection of communications chips, and got the production line after the distribution of the chip can be used to detect production errors; the system uses automated embodiment, instead of doing so operations, improve production efficiency.

\section{Conclusions}

In this paper, the image detection method of photon device line circuit was studied, which can be used in designing process of photon device, and reflect quality of products. The method proposed in this paper can achieve the effect that the device is in line with the design of the circuit.

\section{Acknowledgments}

This work is partially supported by Special Funds for innovative Program of Foshan, Guangdong (no.2014HT100112).Science and Technology Program of Guangzhou, China (no.201510010094).

Science and Technology Planning Project of Guangdong Province, China (no.2013B091500046,no.2013B040500004)

\section{Reference}

[1]S.E.Miller, Integrated Optics: An Introduction, Bell Syst. Tech.J.,1969,vol.48:2059-2068

[2] Sundareswara R, Schrater P R . Bayesian Modeling of Camera Calibration and Reconstruction

[C] //Proceedings of the fifth International Conference on 3D Digital Imaging and Modeling, 2005.

[3]P Viola,M Jones.Rapid Object Detection using a Boosted Cascade of Simple[C]. Computer Vision and Pattern Recognition, Proceedings of the 2001 IEEE Computer Society Conference on, Kauai Marriott, Hawaii, CVPR,2001:511-518

[4]Herbert Bay, Andreas Ess, Tinne Tuytelaars,Luc Van Gool. Speeded-up Robust Features(SURF)[J]. Computer Vision and Image Understanding,2008,110(3):346-359

[5]Kapur J N, Sahoo P K, Wong A K C. A new method for grey-level picture thresholding using the entropy of the histogram[J]. Computer Vision, Graphics, and Image Processing, 1985, 29(3):273-285. 